\title{
Individualized Assessment of Exercise Capacity in Response to Acute and Long-Term Enzyme Replacement Therapy in Pediatric Pompe Disease
}

\author{
Ronen Bar-Yoseph ${ }^{1,+}$, Galit Tal ${ }^{2,+}$, Elena Dumin ${ }^{3}$, Moneera Hanna ${ }^{4}$, Gur Mainzer ${ }^{5}$, Merav Zucker-Toledano ${ }^{6}$, \\ George Shallufi ${ }^{4}$, Mira Jahshan ${ }^{7}$, Hanna Mandel ${ }^{7,8,+}$ and Lea Bentur ${ }^{7,9, *,+}$
}

check for updates

Citation: Bar-Yoseph, R.; Tal, G.; Dumin, E.; Hanna, M.; Mainzer, G.; Zucker-Toledano, M.; Shallufi, G.; Jahshan, M.; Mandel, H.; Bentur, L. Individualized Assessment of Exercise Capacity in Response to Acute and Long-Term Enzyme Replacement Therapy in Pediatric Pompe Disease. J. Pers. Med. 2021, 11, 1105. https://doi.org/10.3390/ jpm11111105

Received: 27 August 2021

Accepted: 22 October 2021

Published: 28 October 2021

Publisher's Note: MDPI stays neutral with regard to jurisdictional claims in published maps and institutional affiliations.

Copyright: (c) 2021 by the authors. Licensee MDPI, Basel, Switzerland. This article is an open access article distributed under the terms and conditions of the Creative Commons Attribution (CC BY) license (https:/ / creativecommons.org/licenses/by/ $4.0 /)$.
1 Ruth Children's Hospital, Pediatric Pulmonary Institute, Technion Faculty of Medicine, Haifa 3109601, Israel; r_bar-yoseph@rambam.health.gov.il

2 Metabolic Unit, Ruth Children's Hospital, Technion Faculty of Medicine, Haifa 3109601, Israel; g_tal@rambam.health.gov.il

3 Metabolic Laboratory Unit, Rambam Health Care Campus, Haifa 3109601, Israel; duminelena@gmail.com

4 Ruth Children's Hospital, Pediatric Pulmonary Institute, Haifa 3109601, Israel; m_hanna@rambam.health.gov.il (M.H.); g_shallufi@outlook.com (G.S.)

5 Pediatric Cardiology, Baruch Padeh Medical Center, Poriya 1528001, Israel; gurmainzer@gmail.com

6 Ruth Children's Hospital, Pediatric Cardiology Institute, Haifa 3109601, Israel; meravzt8@gmail.com

7 Technion Faculty of Medicine, Haifa 3109601, Israel; mira.jahshan@campus.technion.ac.il (M.J.); h_mandel@rambam.health.gov.il (H.M.)

8 Department of Genetics and Metabolic Disorders, Ziv Medical Center, Safed 13100, Israel

9 Children's Hospital, Rambam Health Care Center, Pediatric Pulmonary Institute, Haifa 3109601, Israel

* Correspondence: 1_bentur@rambam.health.gov.il; Tel.: +972-4-777-4360

+ These authors equally contributed to this manuscript.

Abstract: Background: Enzyme replacement therapy (ERT) with alglucosidase alfa improves the prospect of patients with infantile-onset Pompe disease (IOPD). However, a progressive decline has been reported. Objective quantification of the response to ERT when assessing newer strategies is warranted. Methods: This combined retrospective-prospective study assessed the acute and long-term effects of ERT on exercise in IOPD patients. Evaluation included cardiopulmonary exercise testing (CPET), 6-min walking test (6MWT), spirometry, motor function test (GMFM-88) and enzyme blood levels. Results: Thirty-four CPETs (17 pre- and 17 two days-post ERT) over variable follow-up periods were performed in four patients. Two days following ERT, blood enzyme levels increased (median, 1.22 and $10.15 \mu \mathrm{mol} / \mathrm{L} / \mathrm{h}(p=0.003)$ ). However, FEV1, FVC and GMFM-88, the median $6 \mathrm{MWD}$ and the peak $\mathrm{VO}_{2}$ were unchanged. Long-term evaluations showed stabilization in young patients but progressive deterioration in adolescents. Clinical deterioration was associated with more pronounced deterioration in peak $\mathrm{VO}_{2}$ followed in the decreasing order by $6 \mathrm{MWD}, \mathrm{FVC}$ and GMFM-88. Conclusions: The peak $\mathrm{VO}_{2}$ and $6 \mathrm{MWD}$ might serve as more sensitive markers to assess clinical deterioration. More studies are needed to clarify the sensitivity of the peak $\mathrm{VO}_{2}$ and $6 \mathrm{MWT}$ for quantification of individualized response. This may be important when assessing newer strategies and formulations in IOPD.

Keywords: infantile-onset Pompe disease (IOPD); enzyme replacement therapy (ERT); cardiopulmonary exercise testing (CPET); 6 min walking test (6MWT); oxygen uptake at the peak of exercise (peak $\left.\mathrm{VO}_{2}\right)$

\section{Introduction}

Pompe disease is an inherited autosomal recessive glycogen storage disease caused by partial or total deficiency of acid $\alpha$-glucosidase (GAA), resulting in massive accumulation of glycogen in lysosomes of different tissues. Severity of the disease and involvement of various organs are considered to be related to residual GAA activity [1]. In infantile-onset Pompe disease (IOPD), there is complete or near complete loss of GAA activity, and patients present early in life with severe hypotony, hypertrophic cardiomyopathy and early death 
without treatment. Patients with the late-onset disease present with progressive muscle weakness that may lead to loss of mobility and assisted ventilation, but without cardiac involvement [2]. In 2006, ERT with recombinant human GAA (Genzyme Corporation, Cambridge, MA, USA) was approved and since then, decisive modification of the course of the disease has been reported.

Clinical studies in infants have shown that ERT led to complete resolution of cardiomyopathy and improvement in skeletal muscle functions with achievement of independent walking, higher levels of physical activity and survival beyond infancy [3,4]. However, motor skills improvement in infancy is followed by a progressive decline. Different factors are considered to contribute to the clinical course [5], including age at initiation of ERT, extent of the baseline pathology, antibodies formation, cross-reactive immunologic material (CRIM) status [6], as well as explanations related to ERT distribution in skeletal muscle or other pathomechanisms, such as abnormal autophagy.

The multifactorial and heterogeneous response and the clinical deterioration on ERT suggests that enzyme replacement does not keep up with the rate of glycogen accumulation. The possibility that increasing drug delivery by either increasing dosage or frequency of administration or by means of newer formulations might halt the progression of the disease has been suggested. Common evaluation parameters include patient-reported outcome measures (PROMs), motor function scores, pulmonary function tests and walking assessments (e.g., 6 min walking test, 6MWT) for ambulatory patients [7-9].

Outcome parameters that may assess disease progression in newer strategies are needed. Patients' exercise limitations contribute significantly to the patients' perception of impaired well-being. While the focus on metabolic/functional capacity (e.g., cardiopulmonary exercise testing, CPET) in adult late-onset Pompe disease (LOPD) is growing rapidly, the data on pediatric IOPD patients are scarce $[10,11]$. Our aim was to provide a more objective quantification of the acute and long-term effect of ERT on exercise capacity and explore the possible relationship between the blood enzyme level and exercise capacity. Such an evaluation may help to compare disease progression with the use of newer strategies and formulations.

\section{Materials and Methods}

This was a prospective and partially retrospective case-control study assessing patients with IOPD. Diagnosis was confirmed by deficient GAA activity in cultured fibroblasts or by means of ultrahigh-performance liquid chromatography-tandem mass spectrometry (UPLC-MS/MS) in dry blood spots and by means of mutational analysis of genomic DNA. Cross-reactive immunologic material (CRIM) status was determined by means of Western blot analysis in cultured skin fibroblasts or according to the predicted GAA mutation severity.

The study was approved by the institutional review board (IRB-045-18). Written consent was obtained from the pediatric patients (assents) and from their legal guardians (the parents of the minors). Evaluation was carried out in our CPET lab, situated in a tertiary university-affiliated medical center. The inclusion criteria were as follows: Pompe disease, age of 5-18 years, replacement ERT $\geq 1$ year, capable of cycling on a stationary bicycle or walking/running on a treadmill. The exclusion criteria were as follows: invasive ventilation and/or continuous oxygen dependence $24 / 7$ or acute illness on the visit day or one day before.

\subsection{Study Measures}

Each patient underwent evaluation prior to and two days after infusion of GAA ERT (Genzyme) on multiple occasions over a variable follow-up period. Dosing was clinically adjusted and prescribed by the head of our metabolic unit. Evaluation included CPET, 6MWT, motor function score (GMFM-88) and self-collected blood samples (on a Guthrie card) for blood GAA enzyme levels. 
Pre-ERT: The patients arrived at 7:00 a.m., anthropometrics and vital signs (resting heart rate, pulse oximetry and blood pressure) were collected, and a complete neuromuscular evaluation was carried out (gross motor function measure score sheet (GMFM-88), 6MWT, pre-CPET questionnaire (demographics, physical activity level, risk assessment, asthma/atopy/smoking history, family history), pulmonary function tests and CPET. Following evaluation, the patient started infusion of ERT.

Post-ERT: Two days following visit 1, a similar evaluation was performed.

\subsubsection{Spirometry}

Spirometry was performed in accordance with the ATS/ERS (American Thoracic Society/European Respiratory Society) Task Force using a Quark PFT spirometer (Cosmed, Italy) [12]. Measurements of respiratory functions, FVC (forced vital capacity), FEV1 (forced expiratory volume in the first second), FEV1/FVC and FEF25-75 (forced expiratory flow, 25-75\%) and MVV (maximum voluntary ventilation), were recorded [13].

\subsubsection{CPET}

CPET was performed following spirometry and 15 min of rest using a Quark CPET metabolic cart (Cosmed, Rome, Italy) according to the ATS guidelines [14]. All the exercise tests were carried out by the same experienced physician (R.B.-Y.) and the same technician (H.M.) using an ergocycle, beginning with no resistance warm-up lasting 1-3 min followed by incremental increase in resistance adapted to the patient's functional capacity according to the examiner's free judgment and ranging from 5 to 20 Watts/minute (ramp protocol). Those patients who were unable to perform the test on a cycle ergometer were tested on a treadmill with an equivalent incremental protocol. The cardiorespiratory parameters measured included 12-lead ECG, respiration rate (RR), heart rate (HR), oxygen saturation $\left(\mathrm{SpO}_{2}\right)$, blood pressure (BP), tidal volume (TV), minute ventilation (VE) ventilatory reserve, pulmonary oxygen uptake $\left(\mathrm{VO}_{2}\right)$, carbon dioxide flow rejected by the ventilation system $\left(\mathrm{VCO}_{2}\right)$, respiratory equivalents $\left(\mathrm{VE} / \mathrm{VO}_{2}, \mathrm{VE} / \mathrm{VCO}_{2}\right)$, end tidal carbon dioxide $\left(\mathrm{ETCO}_{2}\right)$, anaerobic threshold (AT), oxygen pulse $\left(\mathrm{O}_{2}\right.$ pulse) and respiratory exchange ratio (RER). The criteria for terminating the test were as follows: inability to maintain pedaling cadence $(<60 \mathrm{rpm})$ in association with subjective evidence of fatigue (sweating, hyperpnea) and one or more of the following: peak $\mathrm{VO}_{2}>80 \%$ of the predicted value, maximum heart rate $>80 \%$ of the HR predicted $(\mathrm{HRpred}=208-($ age $\times 0.7))[15,16]$, RER $>1.0$ or reaching the $\mathrm{VO}_{2}$ plateau (failure to increase oxygen uptake despite a continuous increase in work) [17]. Breathing reserve (BR) was calculated as follows: (MVV-peak VE)/MVV; low BR was defined as $\mathrm{BR} \%<15 \%$ or $\mathrm{BR}<11 \mathrm{~L} / \mathrm{min}$ [18].

\subsubsection{Six-Minute Walking Test}

Six-minute walking test was performed according to the American Thoracic Society (ATS) guidelines [19]. $\mathrm{SpO}_{2}, \mathrm{BP}, \mathrm{HR}$ and $\mathrm{RR}$ were evaluated pre- and post-test. As recommended, the patients were instructed to walk as far as possible along a 30-m-long flat corridor for six minutes; $6 \mathrm{MWD}$ was expressed in meters and the age-related percentage predicted $[20,21]$.

\subsubsection{Motor Function}

Motor function: Muscle strength was evaluated by a designated and experienced physiotherapist. Grading was performed according to the gross motor function measure score sheet (GMFM-88) [22]. This is a standardized observational instrument designed and validated to measure change in the gross motor function over time in children. Five dimensions are evaluated: "lying and rolling", "sitting", "crawling and kneeling", "standing" and "walking, running and jumping". The scoring ranges from $0=$ does not initiate, $1=$ initiates, 2 = partially completes, $3=$ completes, $\mathrm{NT}=$ not tested. 


\subsubsection{Evaluation of Alpha-Glucosidase Activity in Dried Blood Spots}

Self-collected blood samples (on a Guthrie card) were requested. Lysosomal GAA was extracted from dried blood spots and incubated with an enzyme-specific cocktail containing the corresponding substrate and the internal standard. This enzyme cocktail was prepared using a commercially available mixture of the substrate and the internal standard at the predetermined optimized molar ratio. After incubation, the enzymatic reaction was quenched; the mixtures of the reaction products were prepared using liquid-liquid extractions and quantified simultaneously using selected ion monitoring on a UPLC-MS/MS system. Mass spectrometric analysis was performed using a tandem mass spectrometer (XEVO TQ-S MICRO, UPLC, Waters Ltd., Manchester, UK) equipped with a UPLC separation module (ACQUITY H CLASS, Waters Ltd., Manchester, UK) with an electrospray ionization source operating in the electrospray-positive mode. Quality control, data processing and analysis were performed according to the good laboratory practice. For each reaction, low (enzyme activity below the cutoff value of $2 \mu \mathrm{mol} / \mathrm{L} / \mathrm{h}$ ) and high (enzyme activity above the cutoff value of $100 \mu \mathrm{mol} / \mathrm{L} / \mathrm{h}$ ) controls from the CDC were used. The blank sample was filter paper without spotted blood.

\subsection{Statistics}

Descriptive analysis was used. The results are expressed as the median and IQR2575. The pre-ERT parameters were compared to the post-ERT ones by means of a nonparametric paired $t$-test. Pearson's correlation was used to assess the correlation between the parameters before and after ERT. A $p$-value lower than 0.05 was assumed as significant. The coprimary efficacy endpoints were CPET and 6MWD.

\section{Results}

Of the 10 IOPD patients followed by the metabolic unit, six patients were excluded (due to continuous mechanical ventilation, oxygen dependence and/or inability to walk/cycle). Hence, only four patients (5-11 years old) were capable of performing repeated evaluation and were included in the analysis. The patients' characteristics are shown in Table 1 . The four patients successfully performed all the measurements (spirometry, GMFM-88, 6MWT, CPET) before and two days after ERT administration on several timepoints. No adverse effects were reported during the study.

Table 1. Demographics and characteristics of the study population at first evaluation.

\begin{tabular}{|c|c|c|c|c|}
\hline & Patient 1 & Patient 2 & Patient 3 & Patient 4 \\
\hline Age (years)/sex & 5.5/Female & 6/Male & 11/Male & 10/Male \\
\hline Study duration (years) & 1.2 & 1.3 & 5.3 & 6 \\
\hline Height $(\mathrm{cm})$ & 112.5 & 113 & 139 & 145 \\
\hline Weight (kg) & 21.2 & 26.5 & 37.2 & 44.1 \\
\hline BMI (\%) & $16.8(83)$ & $20.8(99)$ & $19.3(78)$ & $21(92)$ \\
\hline GAA mutation & R854X/L355P & $\mathrm{L} 355 \mathrm{P} / \mathrm{D} 404 \mathrm{~N}$ & L355P/L355P & L355P/D404N \\
\hline CRIM status & Positive & Positive & Positive & Positive \\
\hline Ethnicity & Muslim & Druze & Druze & Druze \\
\hline Onset & Infantile & Infantile & Infantile & Infantile \\
\hline ERT onset age (months) & 5 & 3 & 7 & 1 \\
\hline Cardiomyopathy at infancy & Yes & Yes & Yes & Yes \\
\hline Current echo & Normal & Normal & Normal & Normal \\
\hline Drop foot & No & Yes & Yes & Yes \\
\hline Gastrostomy & No & Yes & No & No \\
\hline
\end{tabular}

Note: $\mathrm{BMI}=$ body mass index; GAA = acid $\alpha$-glucosidase; $\mathrm{CRIM}=$ cross-reactive immunological, ERT = enzyme replacement therapy.

CPET: The standard Bruce ramp protocol (treadmill) was used for the younger patients $(1,2)$ and the standard $10 \mathrm{~W} / \mathrm{min}$ ramp protocol (cycle ergometer) was used for the older patients $(3,4)$. The criteria for the maximum test were met by all the patients in all the tests. The duration of the test was as expected for a ramp CPET protocol ( $\approx 10 \mathrm{~min})$. Exercise- 
induced bronchoconstriction (drop of $\geq 12 \%$ in FEV1) was not observed on any of the study visits. All the patients reached the target heart rate $(>80 \%$ of the predicted value) at all the CPETs. No drop in oxygen saturation was observed.

\subsection{Paired Evaluation before and Two Days after Enzyme Administration}

A total of 34 (17 pre- and 17 post-ERT) comprehensive evaluations were performed in these four patients. Tables $2-5$ demonstrate the major parameters before and two days after ERT.

Table 2. Patient 1.

\begin{tabular}{|c|c|c|c|c|c|c|}
\hline & \multicolumn{2}{|c|}{ First Evaluation } & \multicolumn{2}{|c|}{$\begin{array}{l}\text { Second Evaluation } \\
\qquad(+7 \text { Months })\end{array}$} & \multicolumn{2}{|c|}{$\begin{array}{l}\text { Third Evaluation } \\
\text { (+14 Months) }\end{array}$} \\
\hline & Post-ERT & Pre-ERT & Post-ERT & Pre-ERT & Post-ERT & Pre-ERT \\
\hline Age at test (years) & 5.5 & 5.5 & 6.2 & 6.2 & 6.7 & 6.7 \\
\hline Height $(\mathrm{cm})$ & 112.5 & 112.5 & 116.5 & 116.5 & 119 & 119 \\
\hline BMI percentile & 83 & 83 & 73 & 73 & 84 & 84 \\
\hline ERT dose (mg/kg) & 40/EOW & $40 / \mathrm{EOW}$ & 40/EOW & $40 / \mathrm{EOW}$ & 40/EOW & $40 / \mathrm{EOW}$ \\
\hline Enzyme level ( $\mu \mathrm{mol} / \mathrm{L} / \mathrm{h})$ & 34.85 & 1.22 & $\mathrm{~N} / \mathrm{A}$ & 1.24 & 49.87 & 0.83 \\
\hline FEV1 (L/s) & 1.12 & 1.07 & 1.22 & 1.17 & 1.4 & 1.29 \\
\hline FEV1 (\% of the predicted value) & 99 & 94 & 100 & 96 & 109 & 101 \\
\hline FVC $(\mathrm{L})$ & 1.19 & 1.42 & 1.26 & 1.25 & 1.46 & 1.34 \\
\hline FVC (\% of the predicted value) & 97 & 115 & 94 & 93 & 103 & 94 \\
\hline Peak HR (bpm) & 198 & 197 & 199 & 195 & 208 & 199 \\
\hline Absolute peak $\mathrm{VO}_{2}(\mathrm{~mL} / \mathrm{min})$ & 615 & 647 & 698 & 626 & 731 & 695 \\
\hline Specific peak $\mathrm{VO}_{2}(\mathrm{~mL} / \mathrm{kg} / \mathrm{min})$ & 29 & 30.5 & 31.6 & 28.3 & 30 & 28.5 \\
\hline Peak $\mathrm{VO}_{2}$ (\% of the predicted value) & 62 & 65 & 68 & 61 & 67 & 64 \\
\hline $\mathrm{VE} / \mathrm{VCO}_{2}$ slope & 38.7 & 37.8 & 41.7 & 32 & 42.4 & 33.5 \\
\hline $\mathrm{O}_{2}$ pulse (\% of the predicted value) & 64 & 68 & 70 & 64 & 65 & 65 \\
\hline Breathing reserve & $\mathrm{L}$ & $\mathrm{N}$ & $\mathrm{L}$ & $\mathrm{N}$ & $\mathrm{L}$ & $\mathrm{N}$ \\
\hline 6MWD (meters) & 495 & 495 & 576 & 527 & 555 & 534 \\
\hline $\begin{array}{c}\text { Walking } \% \text {, running }(\%), \text { jumping }(\%) \\
\text { GMFM-88) }\end{array}$ & 100 & 100 & 100 & 100 & 100 & 100 \\
\hline Total score (\%) (GMFM-88) & 98.5 & 99.6 & 98.4 & 98.8 & 100 & 100 \\
\hline
\end{tabular}

Note: Legends to Tables 2-5: BMI = body mass index, ERT = enzyme replacement therapy; EOW = every other week; FEV1 = forced expiratory volume in the first second; $\mathrm{FVC}=$ forced vital capacity; $\mathrm{HR}=$ heart rate; Peak $\mathrm{VO}_{2}=$ oxygen uptake at the peak of exercise; $\mathrm{VE}=$ minute ventilation; $\mathrm{VCO}_{2}=$ carbon dioxide production; $\mathrm{N}=$ normal; $6 \mathrm{MWD}=6$-min walking distance; $\mathrm{GMFM}-88=$ motor assessment.

Table 3. Patient 2.

\begin{tabular}{|c|c|c|c|c|c|c|}
\hline & \multicolumn{2}{|c|}{ First Evaluation } & \multicolumn{2}{|c|}{$\begin{array}{l}\text { Second Evaluation } \\
\qquad(+7 \text { Months })\end{array}$} & \multicolumn{2}{|c|}{$\begin{array}{l}\text { Third Evaluation } \\
\text { (+15 Months) }\end{array}$} \\
\hline & Post-ERT & Pre-ERT & Post-ERT & Pre-ERT & Post-ERT & Pre-ERT \\
\hline Age at test (years) & 6.2 & 6.2 & 6.9 & 6.9 & 7.5 & 7.5 \\
\hline Height $(\mathrm{cm})$ & 113 & 113 & 118 & 118 & 123 & 123 \\
\hline BMI percentile & 99 & 99 & 97 & 97 & 97 & 97 \\
\hline ERT dose (mg/kg) & 20/EOW & 20/EOW & 40/EOW & $40 / \mathrm{EOW}$ & 40/EOW & $40 / \mathrm{EOW}$ \\
\hline Enzyme level ( $\mu \mathrm{mol} / \mathrm{L} / \mathrm{h})$ & 14.68 & 1.34 & 9.35 & 0.95 & 6.45 & 1.35 \\
\hline FEV1 (L/s) & 1.03 & 1.29 & 1.16 & 1.2 & 1.12 & 1.06 \\
\hline FEV1 ( $\%$ of the predicted value) & 87 & 104 & 88 & 91 & 77 & 73 \\
\hline FVC $(\mathrm{L})$ & 1.15 & 1.47 & 1.33 & 1.38 & 1.32 & 1.24 \\
\hline FVC ( $\%$ of the predicted value) & 87 & 107 & 90 & 94 & 80 & 76 \\
\hline Peak HR (bpm) & 202 & 204 & 190 & 172 & 170 & 185 \\
\hline Absolute peak $\mathrm{VO}_{2}(\mathrm{~mL} / \mathrm{min})$ & 751 & 721 & 726 & 644 & 736 & 590 \\
\hline Specific peak $\mathrm{VO}_{2}(\mathrm{~mL} / \mathrm{kg} / \mathrm{min})$ & 28.3 & 27.2 & 25.9 & 23 & 23.6 & 18.9 \\
\hline Peak $\mathrm{VO}_{2}(\%$ of the predicted value) & 65 & 59 & 56 & 49 & 49 & 40 \\
\hline $\mathrm{VE} / \mathrm{VCO}_{2}$ slope & 39.9 & 32.3 & 40 & 42.4 & 38 & 43.2 \\
\hline $\mathrm{O}_{2}$ pulse ( $\%$ of the predicted value) & 65 & 59 & 60 & 58 & 59 & 43 \\
\hline Breathing reserve & $\mathrm{L}$ & $\mathrm{N}$ & $\mathrm{N}$ & $\mathrm{N}$ & $\mathrm{N}$ & $\mathrm{N}$ \\
\hline 6MWD (meters) & 306 & 306 & 324 & 315 & 288 & 263 \\
\hline $\begin{array}{l}\text { Walking (\%), running (\%), jumping (\%) } \\
\text { (GMFM-88) }\end{array}$ & 54.1 & 58.3 & 61.1 & 54.1 & 50 & 50 \\
\hline Total score (\%) (GMFM-88) & 83.4 & 84.3 & 85.4 & 81.8 & 80.2 & 79.88 \\
\hline
\end{tabular}


Table 4. Patient 3.

\begin{tabular}{|c|c|c|c|c|c|c|c|c|}
\hline & \multicolumn{2}{|c|}{ First Evaluation } & \multicolumn{2}{|c|}{$\begin{array}{l}\text { Second Evaluation } \\
(+1 \text { Month })\end{array}$} & \multicolumn{2}{|c|}{$\begin{array}{l}\text { Third Evaluation } \\
\text { (+55 Months) }\end{array}$} & \multicolumn{2}{|c|}{$\begin{array}{l}\text { Fourth Evaluation } \\
\quad(+63 \text { Months })\end{array}$} \\
\hline & Post-ERT & Pre-ERT & Post-ERT & Pre-ERT & Post-ERT & Pre-ERT & Post-ERT & Pre-ERT \\
\hline Age at test (years) & 11.2 & 11.2 & 11.3 & 11.3 & 15.9 & 15.9 & 16.5 & 16.5 \\
\hline Height $(\mathrm{cm})$ & 139 & 139 & 139 & 139 & 165 & 165 & 166 & 166 \\
\hline BMI percentile & 78 & 78 & 78 & 78 & 65 & 65 & 57 & 57 \\
\hline ERT dose (mg/kg) & 20/EOW & 20/EOW & $40 / \mathrm{EOW}$ & $40 / \mathrm{EOW}$ & $20 / \mathrm{W}$ & $20 / \mathrm{W}$ & $20 / \mathrm{W}$ & $20 / \mathrm{W}$ \\
\hline Enzyme level ( $\mu \mathrm{mol} / \mathrm{L} / \mathrm{h})$ & 8.7 & 0.55 & 9.35 & 2 & $\mathrm{~N} / \mathrm{A}$ & 2.85 & 10.15 & 3.55 \\
\hline FEV1 (L/s) & 1.64 & 1.7 & 1.8 & 1.78 & 2.9 & 2.93 & 2.77 & 2.84 \\
\hline FEV1 ( $\%$ of the predicted value) & 81 & 85 & 89 & 88 & 80 & 80 & 73 & 75 \\
\hline FVC (L) & 1.72 & 1.78 & 2.03 & 1.93 & 2.96 & 2.98 & 2.8 & 3.08 \\
\hline FVC ( $\%$ of the predicted value) & 75 & 78 & 88 & 84 & 71 & 71 & 64 & 71 \\
\hline Peak HR (bpm) & 172 & 166 & 189 & 166 & 175 & 130 & 168 & 157 \\
\hline Absolute peak $\mathrm{VO}_{2}(\mathrm{~mL} / \mathrm{min})$ & 1400 & 1308 & 1610 & 1346 & 1197 & 1117 & 1147 & 1119 \\
\hline Specific peak $\mathrm{VO}_{2}(\mathrm{~mL} / \mathrm{kg} / \mathrm{min})$ & 37.6 & 35.2 & 43.3 & 36.2 & 20.3 & 18.9 & 19.4 & 19 \\
\hline Peak $\mathrm{VO}_{2}$ (\% of the predicted value) & 84 & 79 & 97 & 81 & 43 & 40 & 41 & 40 \\
\hline $\mathrm{VE} / \mathrm{VCO}_{2}$ slope & 37.9 & 24.6 & 41.1 & 30.4 & 36.7 & 23.4 & 32.7 & 32.5 \\
\hline $\mathrm{O}_{2}$ pulse ( $\%$ of the predicted value) & 8.4 & 7.9 & 8.5 & 8.1 & 6.8 & 8.6 & 6.8 & 7.1 \\
\hline Breathing reserve & $\mathrm{N}$ & $\mathrm{N}$ & $\mathrm{L}$ & $\mathrm{N}$ & $\mathrm{N}$ & $\mathrm{N}$ & $\mathrm{N}$ & $\mathrm{N}$ \\
\hline 6MWD (meters) & 620 & 450 & 570 & 580 & 457 & 495 & 468 & 442 \\
\hline $\begin{array}{c}\text { Walking (\%), running (\%), jumping } \\
(\%)(\text { GMFM-88) }\end{array}$ & 98.6 & 97.2 & 100 & 97 & 77.7 & 77.7 & 70.8 & 65.2 \\
\hline Total score (\%) (GMFM-88) & 98 & 97.2 & 97.8 & 97.2 & 89.5 & 89.5 & 91.6 & 87.1 \\
\hline
\end{tabular}

Table 5. Patient 4 .

\begin{tabular}{|c|c|c|c|c|c|c|c|c|c|c|c|c|c|c|}
\hline & \multicolumn{2}{|c|}{$\begin{array}{c}\text { First } \\
\text { Evaluation }\end{array}$} & \multicolumn{2}{|c|}{$\begin{array}{c}\text { Second } \\
\text { Evaluation } \\
(+32 \text { Months })\end{array}$} & \multicolumn{2}{|c|}{$\begin{array}{c}\text { Third } \\
\text { Evaluation } \\
\text { (+33 Months) }\end{array}$} & \multicolumn{2}{|c|}{$\begin{array}{c}\text { Fourth } \\
\text { Evaluation } \\
\text { (+55 Months) }\end{array}$} & \multicolumn{2}{|c|}{$\begin{array}{c}\text { Fifth } \\
\text { Evaluation } \\
\text { (+59 Months) }\end{array}$} & \multicolumn{2}{|c|}{$\begin{array}{c}\text { Sixth } \\
\text { Evaluation } \\
\text { (+62 Months) }\end{array}$} & \multicolumn{2}{|c|}{$\begin{array}{c}\text { Seventh } \\
\text { Evaluation } \\
\text { (+74 Months) }\end{array}$} \\
\hline & $\begin{array}{l}\text { Post- } \\
\text { ERT }\end{array}$ & $\begin{array}{l}\text { Pre- } \\
\text { ERT }\end{array}$ & $\begin{array}{l}\text { Post- } \\
\text { ERT }\end{array}$ & $\begin{array}{l}\text { Pre- } \\
\text { ERT }\end{array}$ & $\begin{array}{l}\text { Post- } \\
\text { ERT }\end{array}$ & $\begin{array}{l}\text { Pre- } \\
\text { ERT }\end{array}$ & $\begin{array}{l}\text { Post- } \\
\text { ERT }\end{array}$ & $\begin{array}{l}\text { Pre- } \\
\text { ERT }\end{array}$ & $\begin{array}{l}\text { Post- } \\
\text { ERT }\end{array}$ & $\begin{array}{l}\text { Pre- } \\
\text { ERT }\end{array}$ & $\begin{array}{l}\text { Post- } \\
\text { ERT }\end{array}$ & $\begin{array}{l}\text { Pre- } \\
\text { ERT }\end{array}$ & $\begin{array}{l}\text { Post- } \\
\text { ERT }\end{array}$ & $\begin{array}{l}\text { Pre- } \\
\text { ERT }\end{array}$ \\
\hline Age at test (years) & 10 & 10 & 12.8 & 12.8 & 12.9 & 12.9 & 14.7 & 14.7 & 15.1 & 15.1 & 15.4 & 15.4 & 16.4 & 16.4 \\
\hline Height $(\mathrm{cm})$ & 145 & 145 & 167 & 167 & 167 & 167 & 177 & 177 & 177 & 178 & 178 & 178 & 186 & 186 \\
\hline BMI percentiles & 92 & 92 & 94 & 92 & 83 & 83 & 97 & 97 & 98 & 98 & 98 & 98 & 95 & 95 \\
\hline ERT dose $(\mathrm{mg} / \mathrm{kg})$ EOW & 20 & 20 & 20 & 20 & 40 & 40 & 40 & 40 & 20 & 20 & 40 & 40 & 40 & 40 \\
\hline Enzyme level ( $\mu \mathrm{mol} / \mathrm{L} / \mathrm{h})$ & $\mathrm{N} / \mathrm{A}$ & $\mathrm{N} / \mathrm{A}$ & 13.4 & 0.85 & $\mathrm{~N} / \mathrm{A}$ & $\mathrm{N} / \mathrm{A}$ & 27.5 & 1.43 & 8.45 & 0.34 & $\mathrm{~N} / \mathrm{A}$ & $\mathrm{N} / \mathrm{A}$ & $\mathrm{N} / \mathrm{A}$ & $\mathrm{N} / \mathrm{A}$ \\
\hline FEV1 (L/s) & 1.99 & 2.01 & 2.51 & 2.29 & 2.74 & 2.62 & 3.09 & 3.12 & 3.3 & 3.41 & 3.39 & 3.23 & 3.45 & 3.45 \\
\hline FEV1 ( $\%$ of the predicted value) & 89 & 90 & 74 & 67 & 80 & 77 & 77 & 78 & 80 & 82 & 81 & 77 & 74 & 74 \\
\hline FVC (L) & 2.44 & 2.38 & 2.82 & 2.73 & 3.1 & 2.95 & 3.69 & 3.44 & 3.65 & 4.01 & 3.85 & 3.8 & 3.94 & 3.95 \\
\hline FVC ( $\%$ of the predicted value) & 95 & 92 & 71 & 69 & 78 & 74 & 78 & 73 & 76 & 82 & 78 & 77 & 72 & 72 \\
\hline Peak HR (bpm) & 190 & 186 & 206 & 200 & 197 & 198 & 203 & 205 & 207 & 204 & 201 & 191 & 198 & 201 \\
\hline Absolute peak $\mathrm{VO}_{2}(\mathrm{~mL} / \mathrm{min})$ & 1764 & 1733 & 2000 & 1908 & 2165 & 1950 & 2320 & 2440 & 2151 & 2274 & 2261 & 2134 & 1838 & 2163 \\
\hline Specific peak $\mathrm{VO}_{2}(\mathrm{~mL} / \mathrm{kg} / \mathrm{min})$ & 40 & 40.2 & 30.3 & 28.9 & 33.31 & 30 & 26.4 & 27.7 & 22.96 & 23.99 & 23.93 & 22.58 & 19.5 & 23.01 \\
\hline Peak $\mathrm{VO}_{2}$ (\% of the predicted value) & 86 & 88 & 63 & 60 & 69 & 62 & 63 & 67 & 59 & 62 & 61 & 58 & 48 & 57 \\
\hline $\mathrm{VE} / \mathrm{VCO}_{2}$ slope & 36 & 31.1 & 30.5 & 29.5 & 26.8 & 28.5 & 29.3 & 27.3 & 31.8 & 30.6 & 28.8 & 30 & 27.9 & 28.4 \\
\hline $\mathrm{O}_{2}$ pulse (\% of the predicted value) & 91 & 95 & 61 & 60 & 70 & 63 & 69 & 71 & 56 & 60 & 60 & 60 & 48 & 56 \\
\hline Breathing reserve & $\mathrm{N}$ & $\mathrm{N}$ & $\mathrm{N}$ & $\mathrm{N}$ & $\mathrm{N}$ & $\mathrm{N}$ & $\mathrm{N}$ & $\mathrm{N}$ & $\mathrm{N}$ & $\mathrm{N}$ & $\mathrm{N}$ & $\mathrm{N}$ & $\mathrm{N}$ & $\mathrm{N}$ \\
\hline 6MWD (meters) & 635 & 660 & 550 & 564 & 554 & 504 & 549 & 531 & 531 & 522 & 508 & 508 & 504 & 496 \\
\hline $\begin{array}{c}\text { Walking (\%), running (\%), jumping } \\
(\%) \text { (GMFM-88) }\end{array}$ & 100 & 100 & 95.8 & 94.4 & 98.6 & 97.2 & 98.6 & 93 & 94.4 & 93 & 90.2 & 90.2 & 91.6 & 87.5 \\
\hline Total Score \%-GMFM-88 & 100 & 100 & 96.58 & 96.9 & 98.2 & 96.58 & 98.1 & 97.5 & 97.84 & 97.56 & 95.9 & 96.5 & 97.2 & 95.4 \\
\hline
\end{tabular}

Analysis of the paired blood enzyme activities showed a significant increase from the median $1.22 \mu \mathrm{mol} / \mathrm{L} / \mathrm{h}(\mathrm{IQR} 25-75,0.83-1.43 \mu \mathrm{mol} / \mathrm{L} / \mathrm{h})$ to the median $10.15 \mu \mathrm{mol} / \mathrm{L} / \mathrm{h}$ (IQR25-75, 8.7-27.5 $\mu \mathrm{mol} / \mathrm{L} / \mathrm{h}$ ) two days after enzyme administration; $p=0.003$.

Pulmonary function tests (FEV1, FVC) showed no significant increase two days postERT (FEV1 median, 82\% (IQR25-75, 76-94\%) and 81\% of the predicted value (IQR25-75, 77-89\%); FVC median, 78\% (IQR25-75, 73-94\%) and 78\% of the predicted value (IQR25-75, $74-92 \%) ; p=0.38$ and $p=0.82$, respectively). The median 6MWD was $504 \mathrm{~m}$ pre-ERT (IQR25-75, 446-532.5 $\mathrm{m}$ ) and $531 \mathrm{~m}$ (IQR25-75, 462.5-562.5 m) post-ERT, $p=0.13$. There was a nonsignificant increase in the peak $\mathrm{VO}_{2}$ (median, from $61 \%$ of the predicted value pre-ERT (IQR25-75, 53-66\%) to 63\% (IQR25-75, 53-66\%) post-ERT; $p=0.063$ ). 


\subsection{Long-Term Effect of ERT}

The repeated measurements for each patient over the study period are shown in Tables 2-5. The individualized patterns of the main outcome parameters (enzyme levels, GMFM-88, 6MWT, $\mathrm{VO}_{2}$ ) are presented in Figures 1-4.

\section{Patient 1 (5.5-6.5 y/o)}

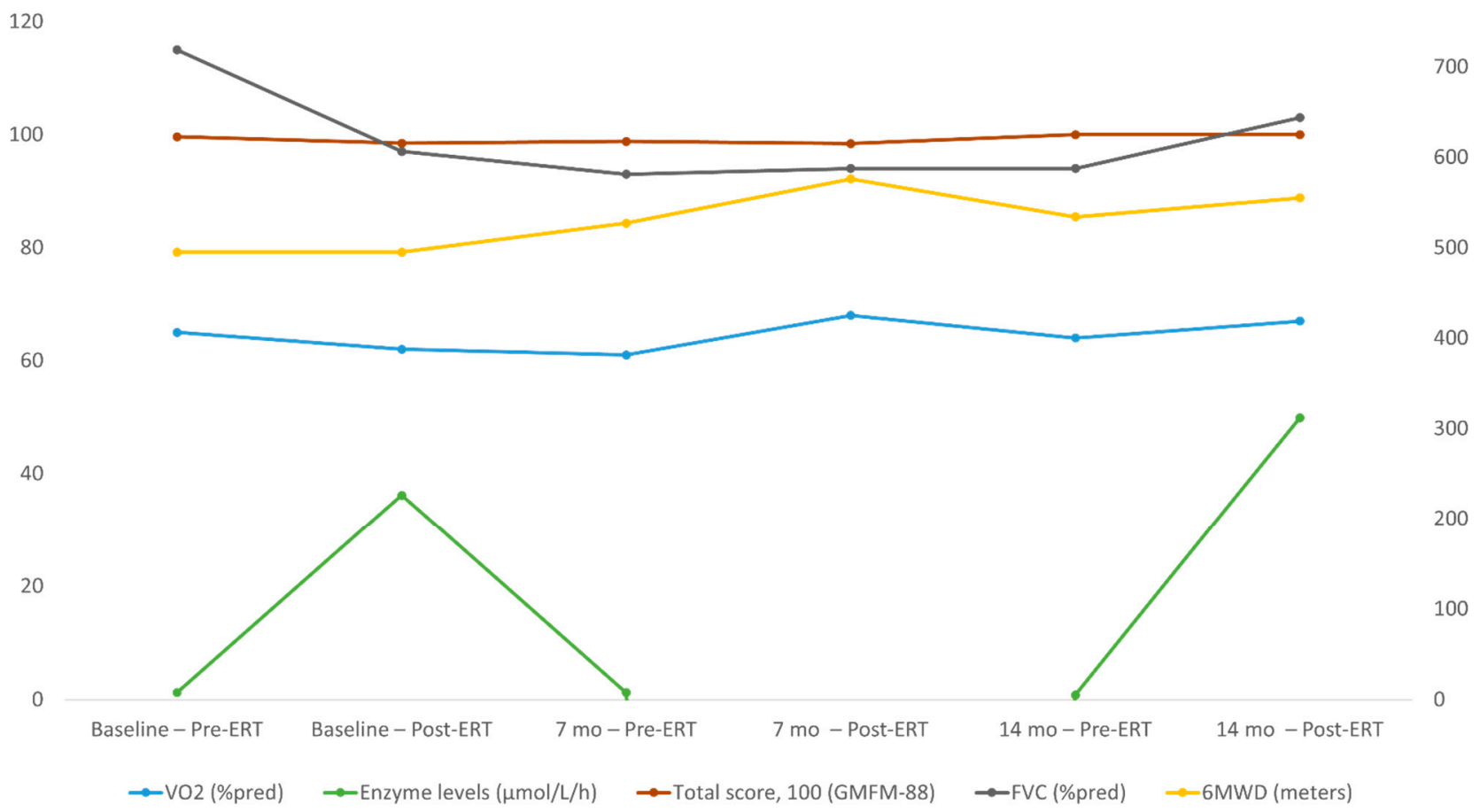

Figure 1. Long-term follow-up of the study parameters (patient 1 ). $y / o=$ year old, $\mathrm{VO}_{2}=$ peak oxygen uptake at the peak of exercise; $\%$ pred $=\%$ of the predicted value; $\mathrm{FVC}=$ forced vital capacity; $6 \mathrm{MWD}=6$-min walking distance.

\section{Patient 2 (6-7 y/o)}
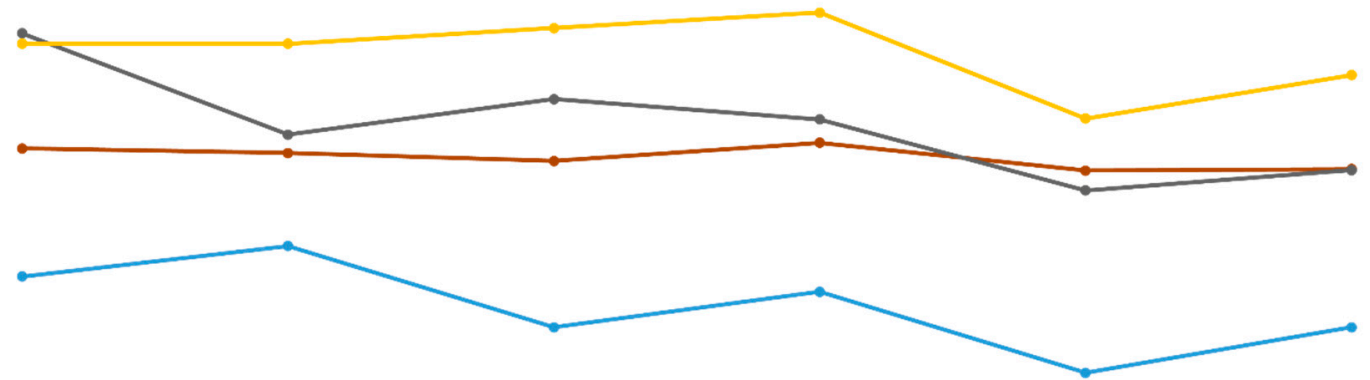

20

0

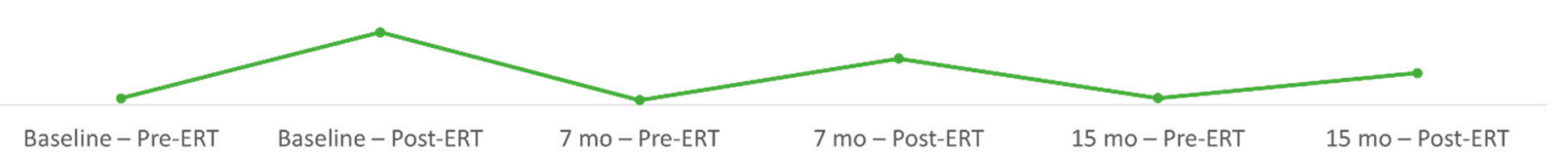

$\rightarrow$ VO2 (\%pred) $\rightarrow$ Enzyme levels ( $\mu$ mol/L/h) $\rightarrow$-Total Score, 100 (GMFM-88) $\rightarrow$ FVC (\%pred) $\rightarrow$ 6MWD (meters)

Figure 2. Long-term follow-up of the study parameters (patient 2). y/o = year old; $\mathrm{VO}_{2}=$ peak oxygen uptake at the peak of exercise; $\%$ pred $=\%$ of the predicted value; $\mathrm{FVC}=$ forced vital capacity; $6 \mathrm{MWD}=6$-min walking distance. 


\section{Patient $3(11-16.5$ y/o)}

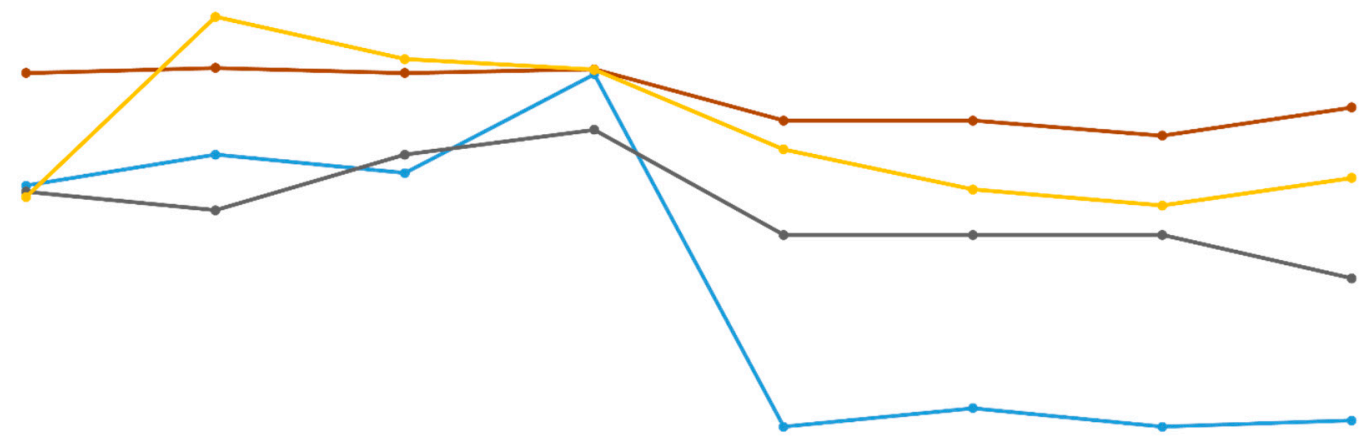

80

60

0

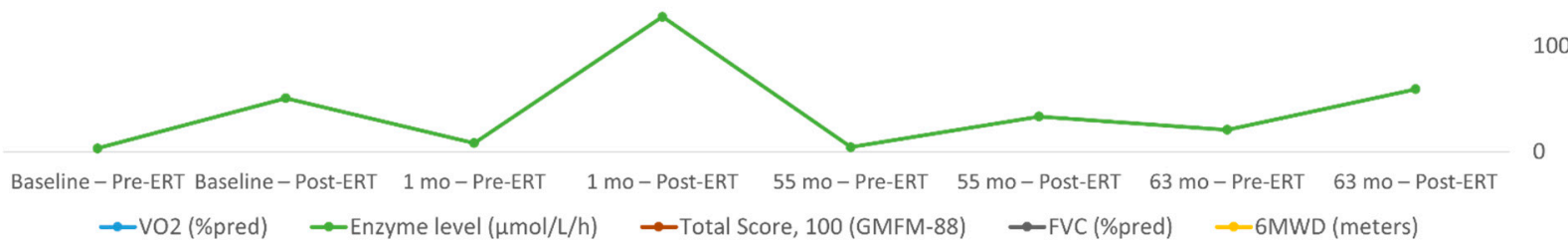

Figure 3. Long-term follow-up of the study parameters (patient 3). $\mathrm{y} / \mathrm{o}=$ year old; $\mathrm{VO}_{2}=$ peak oxygen uptake at the peak of exercise; $\%$ pred $=\%$ of the predicted value; $\mathrm{FVC}=$ forced vital capacity; $6 \mathrm{MWD}=6$-min walking distance.

\section{Patient 4 (10.0-16.4 y/o)}

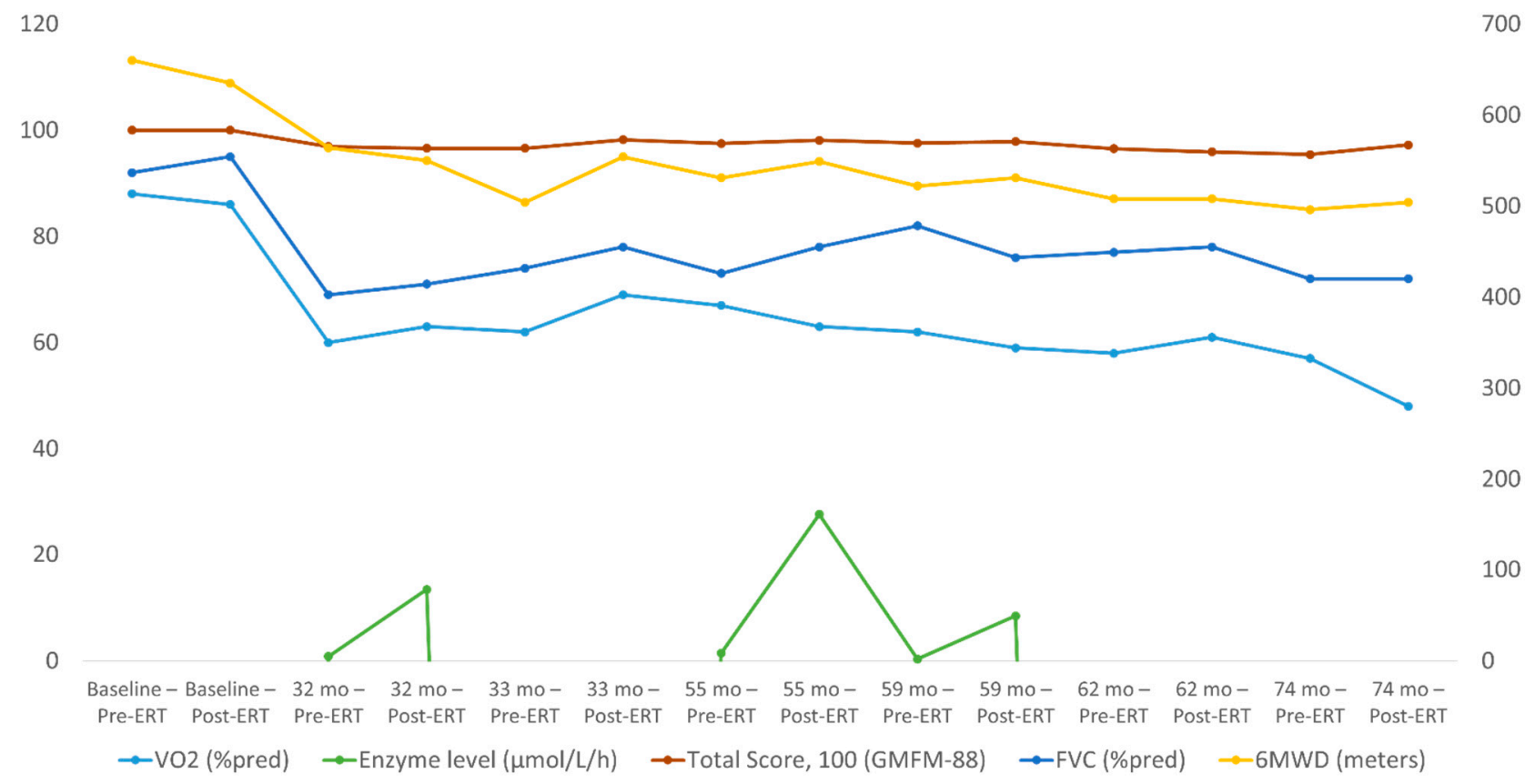

Figure 4. Long-term follow-up of the study parameters (patient 4). $\mathrm{y} / \mathrm{o}=$ year old, $\mathrm{VO}_{2}=$ peak oxygen uptake at the peak of exercise; $\%$ pred $=\%$ of the predicted value; $\mathrm{FVC}=$ forced vital capacity; $6 \mathrm{MWD}=6$-min walking distance.

Patient No. 1 (Table 2, Figure 1) started evaluation at the age of 5.5 years and had six evaluations (three measurements before ERT and three after). At the time of her first evaluation and throughout the study, she was seemingly healthy, with normal developmental motor skills and only minor abnormal findings on her physical examination, suggestive of myopathy. At the baseline, all the parameters evaluated were within the normal range 
except for the peak $\mathrm{VO}_{2}$. Over 14 months, FVC increased from $97 \%$ to $103 \%$ of the predicted value, GMFM-88 increased by 1.5 points (from 98.5 to 100), 6MWD increased by $60 \mathrm{~m}$ (from 495 to $555 \mathrm{~m}$; from $104 \%$ to $108 \%$ of the predicted value) and the peak $\mathrm{VO}_{2}$ increased from $62 \%$ to $67 \%$ of the predicted value; from 29.0 to $30.0 \mathrm{~mL} / \mathrm{kg} / \mathrm{min}$.

Patient No. 2 (Table 3, Figure 2) started evaluation at the age of 6.2 years and had six evaluations (three measurements before ERT and three after). This boy had a waddling gate and delayed gross motor development. He remained clinically stable during the study period. At the baseline, he had normal pulmonary function test results with reduced GMFM-88, 6MWT and peak $\mathrm{VO}_{2}$. Over 15 months, FVC decreased from $87 \%$ to $80 \%$ of the predicted value, GMFM-88 decreased by 3.2 points (from 83.4 to 80.2 ), 6MWD decreased by $18 \mathrm{~m}$ (from 306 to $288 \mathrm{~m}$; from $59 \%$ to $52 \%$ of the predicted value) and the peak $\mathrm{VO}_{2}$ decreased from $65 \%$ to $49 \%$ of the predicted value; from 28.3 to $23.6 \mathrm{~mL} / \mathrm{kg} / \mathrm{min}$.

Patient No. 3 (Table 4, Figure 3) started evaluation at the age of 11.2 years and had eight evaluations (four measurements before ERT and four after). At the time of his first assessment, this patient was seemingly asymptomatic. However, in the previous several years, there was a constant decline in motor skills and complications of peripheral neuropathy. During the last four assessments, he was able to walk only short distances and required a wheelchair for longer distances. At the baseline, he had mildly reduced pulmonary function tests, $6 \mathrm{MWT}$ and peak $\mathrm{VO}_{2}$ and normal GMFM-88. Over 63 months, FVC decreased from $75 \%$ to $64 \%$ of the predicted value, GMFM- 88 decreased by 6.4 points (from 98.0 to 91.6), 6MWD decreased by $152 \mathrm{~m}$ (from 620 to $468 \mathrm{~m}$; from $99 \%$ to $86 \%$ of the predicted value) and the peak $\mathrm{VO}_{2}$ decreased from $84 \%$ to $41 \%$ of the predicted value; from 28.9 to $19.4 \mathrm{~mL} / \mathrm{kg} / \mathrm{min}$.

Patient No. 4 (Table 5, Figure 4) started evaluation at the age of 10.0 years and had 14 evaluations (seven measurements before ERT and seven after). At the time of his first assessments, this patient was also seemingly asymptomatic. However, in the previous several years, there was a slow decline in his motor skills and complications of peripheral neuropathy. He was still mobile and did not require any support. At the baseline, all the parameters evaluated were within the normal range. Over 74 months, FVC decreased from $95 \%$ to $72 \%$ of the predicted value, GMFM-88 decreased by 2.8 points (from 100 to 97.2 ), 6MWD decreased by $131 \mathrm{~m}$ (from 635 to $504 \mathrm{~m}$; from 100\% to $92 \%$ of the predicted value) and the peak $\mathrm{VO}_{2}$ decreased from $86 \%$ to $48 \%$; from 40.0 to $19.5 \mathrm{~mL} / \mathrm{kg} / \mathrm{min}$.

\section{Discussion}

In this study, we evaluated both acute and long-term functional capacity in response to GAA ERT in pediatric IOPD patients. Thirty-four CPET evaluations were performed (17 before and 17 two days after ERT) over variable follow-up times (14-74 months) in the patients receiving ERT since infancy. At the baseline, three of the four patients already had a decrease in oxygen uptake (peak $\mathrm{VO}_{2}$ ), while most of the other parameters were mildly reduced or unaffected. The acute response (two days after infusion of GAA ERT) showed increased blood enzyme levels but no significant changes in FEV1, FVC, GMFM-88, $6 \mathrm{MWD}$ or the median peak $\mathrm{VO}_{2}$. Long-term evaluations showed an improvement and stabilization in young patients but progressive time- and age-related clinical deterioration. The most pronounced deterioration was in the peak $\mathrm{VO}_{2}$, followed in the decreasing order by $6 \mathrm{MWD}, \mathrm{FVC}$ and GMFM-88. This observation suggests that the peak $\mathrm{VO}_{2}$ and $6 \mathrm{MWD}$ might serve as more sensitive markers to assess clinical deterioration, and this finding may be important, especially when assessing newer strategies and formulations.

IOPD is a very rare disease [23]; of the 10 patients followed at our center, only four were able to perform repeated evaluations. All the patients were diagnosed with CRIMpositive IOPD at infancy, had cardiomyopathy at presentation and started ERT between the age of 1-7 months. ERT since infancy led to resolution of cardiomyopathy, respiratory and skeletal muscle functions improvement, achievement of independent walking and survival beyond infancy. The four patients completed their first evaluations at the prepubertal stage. 
In our study, the older patients received GAA ERT for a period of 16 years, the younger ones-for 6 years.

A high degree of variability was observed among the patients in their responses to treatment. A recent report described long-term outcomes in 14 IOPD CRIM-positive patients and showed that ERT maintained the respiratory function in a subgroup of patients, whereas others had a steady decline [7].

The acute and long-term follow-up of patients on ERT is necessary to better understand the variable response and the challenges that hinder its effectiveness and explore the unmet needs.

Objective quantification of the response to ERT, especially when assessing newer strategies and formulations, is warranted. Exercise capacity significantly affects Pompe disease patients' clinical picture and quality of life. CPET outcome may potentially be research and clinical functional assessment of ERT.

The reported data on the acute and long-term effects of ERT on CPET parameters is limited, especially in IOPD. The safety of exercise and CPET was demonstrated in LOPD $[10,24]$, and we recently demonstrated the feasibility and safety of CPET in five pediatric Pompe disease patients [11].

Baseline parameters: At the first assessment, only one patient had mildly reduced FCV, one had reduced motor score and one had reduced $6 \mathrm{MWD}$; this may reflect the change in the natural course of IOPD under early administration of ERT. A recent multicenter study from India (with limited access to ERT) [25] showed improved outcomes and survival in patients receiving ERT compared to those not receiving ERT. However, three of the four patients already had a decrease in oxygen uptake (peak $\mathrm{VO}_{2}$ ), which represents one of the main indices of exercise capacity. This may imply that the peripheral muscles may be more prominent in determining the peak $\mathrm{VO}_{2}$ decrease than the cardiorespiratory component in IOPD; the peak $\mathrm{VO}_{2}$ might be more sensitive than the pulmonary function, GMFM-88 and 6MWT when evaluating clinical status in IOPD.

Acute response to ERT: In clinical practice, Pompe disease patients treated for a long time with ERT often report a subjective improvement of general well-being and reduced fatigability following administration, with a decline toward the end of the two-week interval between infusions.

When we assessed the response two days after ERT, all the patients reported subjective improvement, which correlates with increased enzyme activity measured in DBS. However, this improvement was not supported by either motor assessment or pulmonary function tests. There was a nonsignificant increase in $6 \mathrm{MWD}$ and the peak $\mathrm{VO}_{2}$. We are aware of one similar study assessing the acute effect (one day before and after ERT) on CPET parameters and blood enzyme levels in 11 LOPD patients [26]. Similarly, to this study, no significant differences in the variables related to exercise tolerance were found despite a significant increase in GAA activity in peripheral lymphocytes.

This may imply that these parameters are not sufficiently sensitive to assess individualized acute response to ERT. The small sample size may have resulted in a type 2 error. Alternatively, the increase in blood enzyme levels following therapy may have affected other unmeasured parameters (e.g., the quality-of-life questionnaire, smart watches assessing the activity).

Long-term response to ERT: Two small case series of LOPD assessed the effect of ERT on repeated CPET measures [10,24]. Marzorati et al. demonstrated the positive effect of ERT on some physiological variables associated with exercise tolerance in four patients with LOPD after one year of therapy [24]. Crescimanno et al. evaluated eight LOPD patients by means of CPETs, but only four of them (one-naïve to ERT) were followed up after 36 months of treatment.

Three patients showed a reduced exercise capacity as evaluated by peak oxygen uptake $\left(\mathrm{VO}_{2}\right)$ measured at the CPET and 6MWT at the baseline. At the follow-up, 9.5\% improvement in oxygen uptake and $5.6 \%(25 \mathrm{~m})$ improvement in 6MWD was reported. The difference may be related to different type of disease (late vs. infantile onset), duration and 
dose of ERT used, response variability and sample size [10]. A case study of a 50-year-old female with Pompe disease showed improvement in CPETs after one month of ERT and no further changes following 24 months of ERT [27]. We are unaware of studies repeating CPET measurements over a long-term follow-up period in IOPD. In our study, the two younger patients (patients 1 and 2) remained stable over 14-15 months of follow-up. The two older patients (patients 3 and 4) showed relative stability at their first evaluations. However, when reassessed during puberty and at a post-pubertal stage, they had started to deteriorate. Patients 2-4 are CRIM positive and did not receive any immune suppressant protocol. Patient 1 is compound heterozygote including a severe mutation R854X and the CRIM positive L355P. She received a single course of three consecutive daily methotrexate injections; first injection was given just before the very first Myozyme infusion. None of the patients had significant neutralizing antibody levels. Two patients had zero levels (\#3, $\# 4$ ), one patient (\#1) had antibody titer of 1600 and one patient (\#2) had 1800. Levels of $\geq 6400$ are considered significant [28], therefore, the waning efficacy cannot be attributed to antibodies formation.

The active functional evaluations (e.g., 6MWT, CPET) seemed to better correlate with the reported clinical deterioration. The $6 \mathrm{MWT}$ is a relatively common method to assess progression in Pompe disease and other neuromuscular disorders. However, $\mathrm{VO}_{2}$ peak was affected more than 6MWT, suggesting that it is a more sensitive parameter to evaluate individualized response.

Similarly, Sechi et al. suggested that $\mathrm{VO}_{2}$ peak might be more sensitive than $6 \mathrm{MWT}$ in estimating exercise tolerance in LOPD. The decline in exercise capacity was not explained by overt cardiac or respiratory limitations and probably is related to muscle weakness and deconditioning. Motor assessment by our experienced physiotherapist showed a lower correlation to clinical deterioration, even when specific functional dimensions (e.g., walking, running \& jumping) were measured (Tables 2-5). This suggests that other measures of muscles functions (e.g., MRI, biopsy) should be used.

Indeed, ERT does not cure the disease and the majority of IOPD infants develop progressive myopathy in subsequent years, despite very early initiation of treatment. The most apparent limitation of ERT is the poor response of skeletal muscles with accumulation of glycogen-filled autophagosomes [29]. In LOPD, ERT resulted in stabilization or slowing of progression of the disease. A large double-blind placebo-controlled study carried out in 90 adult patients demonstrated improved walking distance and stabilization of the pulmonary function over an 18-month period [2]; the largest response was seen during the first 2-3 years of treatment [30].

The clinical deterioration observed in IOPD patients on ERT suggests enzyme replacement does not keep up with the rate of glycogen accumulation, and significant unmet medical needs remain. Despite long-term and regular administration of ERT, there is histological evidence of pronounced autophagic vacuoles in skeletal muscle specimens but only a small amount of glycogen storage in lysosomes [29]. This finding could explain in part the progressively reduced effect of ERT, resulting in motor deterioration in these patients [29]. The possibility that increasing drug delivery by either increasing dosage or frequency of administration might halt the progression of the disease was, therefore, suggested.

In our study, two days following ERT, significantly increased blood enzyme levels were revealed (median, $1.22 \mu \mathrm{mol} / \mathrm{L} / \mathrm{h}$ and $10.15 \mu \mathrm{mol} / \mathrm{L} / \mathrm{h} ; p=0.003$ ). Enzyme levels in response to ERT are rarely reported [26]. It should be acknowledged that GAA activity measured in peripheral blood does not necessarily reflect the levels of activity achieved in skeletal muscles. However, determination of GAA activity in muscle tissues would require a muscle biopsy, which was not carried out in this study for ethical reasons.

Few studies have reported on the effect of increasing ERT dosage on the efficacy of treatment [29]. One could speculate that higher enzyme levels may escape autophagy. The preliminary data of a recent study which compared the safety and efficacy of treatment with $40 \mathrm{mg} / \mathrm{kg}$ / week to that of $20 \mathrm{mg} / \mathrm{kg}$ / every other week (EOW) showed that a higher dose leads to improvement in Pompe disease patients [31]. In this study, the dose was prescribed 
and changed by the head of the metabolic unit. The initial ERT dosage in patients 3 and 4 was $20 \mathrm{mg} / \mathrm{kg}$ EOW as recommended by results of the studies performed in 2003-2006 [4]. During the recent years, their dose was increased to $40 \mathrm{mg} / \mathrm{kg}$ EOW or $20 \mathrm{mg} / \mathrm{kg}$ EW (patient 3) due to the policy change in Israel based on results of several studies which recommended high-dose ERT, $40 \mathrm{mg} / \mathrm{kg}$ EOW [32,33]. Patients 1 and 2 had been receiving this higher dose since starting therapy. The low number of patients involved in the study, the different ERT dosages at variable times and the low number of self-collected blood samples precluded further analysis.

Limitations: The major limitation, as in other orphan diseases, is the small sample size. Some of the measurements were performed for clinical purposes before the study was designed, therefore the study is partially retrospective. All the patients started ERT in infancy as clinically indicated, however, with different doses and frequencies. We did not have baseline exercise capacity before GAA ERT initiation. Therefore, the effect of ERT in naïve IOPD could not be evaluated. Inspiratory/expiratory muscle strength (MIP, MEP) tests and health-related quality of life were not assessed. GAA blood enzyme levels were not collected at each visit, hence the response to different doses could not be assessed.

\section{Conclusions}

Individualized assessment of the peak $\mathrm{VO}_{2}$ by means of CPETs may provide a more sensitive assessment of clinical severity and long-term response to treatment.

CPET parameters may be less sensitive to assess the acute effect of ERT in non-naïve ERT patients; 6MWT may provide helpful information in regions with limited access to CPET; larger, long-term prospective studies are needed to clarify the sensitivity of the peak $\mathrm{VO}_{2}$ and 6MWT for quantification of individualized response and the possible relation to blood levels. This may aid in evaluation of different ERT dosages and newer formulations.

Author Contributions: Conceptualization, L.B., H.M. and R.B.-Y.; data curation, R.B.-Y., M.H., G.M., M.Z.-T., E.D., G.T., G.S. and M.J.; formal analysis, R.B.-Y., L.B., G.T., E.D., M.H., G.M., M.Z.-T., G.S. and M.J.; methodology, R.B.-Y., L.B., G.T., E.D. and M.H.; project administration, R.B.-Y., M.H. and G.T.; resources, L.B., H.M. and R.B.-Y.; visualization, R.B.-Y.; writing-original draft preparation, L.B. and R.B.-Y.; writing - review and editing, L.B., R.B.-Y., H.M. and G.T. All authors have read and agreed to the published version of the manuscript.

Funding: This work was supported by an investigator-initiated grant from Sanofi Genzyme. Sanofi Genzyme had no involvement in the collection, analysis and interpretation of data; in the writing of the report; and in the decision to submit the article for publication.

Institutional Review Board Statement: The study was conducted according to the guidelines of the Declaration of Helsinki and approved by the Institutional Review Board of the Rambam Medical Center (protocol code 045-18-RMB, 24 June 2018).

Informed Consent Statement: Informed consent was obtained from all the pediatric patients (assents) and from their legal guardians (the parents of the minors). Clinical trial registration number NCT04755751.

Data Availability Statement: The data presented in this study are available from the corresponding author upon reasonable request.

Conflicts of Interest: Hanna Mandel received in the past an honorarium from Genzyme and is a member of the Pompe Disease Registry Advisory Board for Sanofi Genzyme. Galit Tal received travel grant support from Genzyme. All the other authors declared no conflict of interest.

\section{References}

1. Van der Ploeg, A.T.; Reuser, A.J. Pompe's disease. Lancet 2008, 372, 1342-1353. [CrossRef]

2. Van der Ploeg, A.T.; Clemens, P.R.; Corzo, D.; Escolar, D.M.; Florence, J.; Groeneveld, G.J.; Herson, S.; Kishnani, P.S.; Laforet, P.; Lake, S.L.; et al. A Randomized Study of Alglucosidase Alfa in Late-Onset Pompe's Disease. N. Engl. J. Med. 2010, 362, 1396-1406. [CrossRef]

3. Kishnani, P.S.; Howell, R.R. Pompe disease in infants and children. Proc. J. Pediatr. 2004, 144, S35-S43. [CrossRef] [PubMed] 
4. Kishnani, P.S.; Corzo, D.; Nicolino, M.; Byrne, B.; Mandel, H.; Hwu, W.L.; Leslie, N.; Levine, J.; Spencer, C.; McDonald, M.; et al. Recombinant human acid $\alpha$-glucosidase: Major clinical benefits in infantile-onset Pompe disease. Neurology 2007, 68, 99-109. [CrossRef]

5. Do, H.V.; Khanna, R.; Gotschall, R. Challenges in treating Pompe disease: An industry perspective. Ann. Transl. Med. 2019, 7, 291. [CrossRef]

6. Kishnani, P.S.; Goldenberg, P.C.; DeArmey, S.L.; Heller, J.; Benjamin, D.; Young, S.; Bali, D.; Smith, S.A.; Li, J.S.; Mandel, H.; et al. Cross-reactive immunologic material status affects treatment outcomes in Pompe disease infants. Mol. Genet. Metab. 2010, 99, 26-33. [CrossRef]

7. ElMallah, M.; Desai, A.; Nading, E.; DeArmey, S.; Kravitz, R.; Kishnani, P. Pulmonary outcome measures in long-term survivors of infantile Pompe disease on enzyme replacement therapy: A case series. Pediatr. Pulmonol. 2020, 55, 674-681. [CrossRef]

8. Angelini, C.; Semplicini, C.; Ravaglia, S.; Moggio, M.; Comi, G.P.; Musumeci, O.; Pegoraro, E.; Tonin, P.; Filosto, M.; Servidei, S.; et al. New motor outcome function measures in evaluation of Late-Onset Pompe disease before and after enzyme replacement therapy. Muscle Nerve 2012, 45, 831-834. [CrossRef]

9. Yuan, M.; Andrinopoulou, E.; Kruijshaar, M.; Lika, A.; Harlaar, L.; van der Ploeg, A.; Rizopoulos, D.; van der Beek, N. Positive association between physical outcomes and patient-reported outcomes in late-onset Pompe disease: A cross sectional study. Orphanet J. Rare Dis. 2020, 15, 232. [CrossRef] [PubMed]

10. Crescimanno, G.; Modica, R.; Lo Mauro, R.; Musumeci, O.; Toscano, A.; Marrone, O. Role of the cardio-pulmonary exercise test and six-minute walking test in the evaluation of exercise performance in patients with late-onset Pompe disease. Neuromuscul. Disord. 2015, 25, 542-547. [CrossRef]

11. Bar-Yoseph, R.; Mandel, H.; Mainzer, G.; Gur, M.; Tal, G.; Shalloufeh, G.; Bentur, L. Cardiopulmonary exercise test to quantify enzyme replacement response in pediatric Pompe disease. Pediatr. Pulmonol. 2018, 53, 366-373. [CrossRef] [PubMed]

12. Miller, M.R.; Hankinson, J.; Brusasco, V.; Burgos, F.; Casaburi, R.; Coates, A.; Crapo, R.; Enright, P.; van der Grinten, C.P.M.; Gustafsson, P.; et al. Standardisation of spirometry. Eur. Respir. J. 2005, 26, 319-338. [CrossRef]

13. Quanjer, P.H.; Borsboom, G.J.J.M.; Brunekreef, B.; Zach, M.; Forche, G.; Cotes, J.E.; Sanchis, J.; Paoletti, P. Spirometric reference values for white European children and adolescents: Polgar revisited. Pediatr. Pulmonol. 1995, 19, 135-142. [CrossRef]

14. Ross, R.M.; Beck, K.C.; Casaburi, R.; Johnson, B.D.; Marciniuk, D.D.; Wagner, P.D.; Weisman, I.M. ATS/ACCP Statement on Cardiopulmonary Exercise Testing (multiple letters). Am. J. Respir. Crit. Care Med. 2003, 167, 1451. [CrossRef]

15. Mahon, A.; Marjerrison, A.; Lee, J.; Woodruff, M.; Hanna, L. Evaluating the prediction of maximal heart rate in children and adolescents. Res. Q. Exerc. Sport 2010, 81, 466-471. [CrossRef]

16. Machado, F.; Denadai, B. Validity of maximum heart rate prediction equations for children and adolescents. Arq. Bras. Cardiol. 2011, 97, 136-140. [CrossRef]

17. Rowland, T.; Hagenbuch, S.; Pober, D.; Garrison, A. Exercise tolerance and thermoregulatory responses during cycling in boys and men. Med. Sci. Sports Exerc. 2008, 40, 282-287. [CrossRef]

18. Wasserman, K.; Hansen, J.E.; Sue, D.Y.; Stringer, W.W.; Sietsema, K.E.; Sun, X.G.; Whipp, B.J. Principles of Exercise Testing and Interpretation, 5th ed.; Lippincott Williams \& Wilkins: Philadelphia, PA, USA, 2012.

19. Crapo, R.O.; Casaburi, R.; Coates, A.L.; Enright, P.L.; MacIntyre, N.R.; McKay, R.T.; Johnson, D.; Wanger, J.S.; Zeballos, R.J.; Bittner, V.; et al. ATS statement: Guidelines for the six-minute walk test. Am. J. Respir. Crit. Care Med. 2002, 166, 111-117. [CrossRef]

20. Goemans, N.; Klingels, K.; Van Den Hauwe, M.; Boons, S.; Verstraete, L.; Peeters, C.; Feys, H.; Buyse, G. Six-minute walk test: Reference values and prediction equation in healthy boys aged 5 to12 years. PLoS ONE 2013, 8, e84120. [CrossRef]

21. Kanburoglu, M.K.; Ozdemir, F.M.; Ozkan, S.; Tunaoglu, F.S. Reference Values of the 6-Minute Walk Test in Healthy Turkish Children and Adolescents Between 11 and 18 Years of Age. Respir. Care 2014, 59, 1369-1375. [CrossRef] [PubMed]

22. Lundkvist Josenby, A.; Jarnlo, G.-B.; Gummesson, C.; Nordmark, E. Longitudinal Construct Validity of the GMFM-88 Total Score and Goal Total Score and the GMFM-66 Score in a 5-Year Follow-up Study. Phys. Ther. 2009, 89, 342-350. [CrossRef] [PubMed]

23. Richardson, J.; Kemper, A.; Grosse, S.; Lam, W.; Rose, A.; Ahmad, A.; Gebremariam, A.; Prosser, L. Health and economic outcomes of newborn screening for infantile-onset Pompe disease. Genet. Med. 2021, 23, 758-766. [CrossRef]

24. Marzorati, M.; Porcelli, S.; Bellistri, G.; Morandi, L.; Grassi, B. Exercise testing in late-onset glycogen storage disease type II patients undergoing enzyme replacement therapy. Neuromuscul. Disord. 2012, 22, S230-S234. [CrossRef]

25. Gupta, N.; Kazi, Z.B.; Nampoothiri, S.; Jagdeesh, S.; Kabra, M.; Puri, R.D.; Muranjan, M.; Kalaivani, M.; Rehder, C.; Bali, D.; et al. Clinical and Molecular Disease Spectrum and Outcomes in Patients with Infantile-Onset Pompe Disease. J. Pediatr. 2020, 216, 44-50.e5. [CrossRef] [PubMed]

26. Sechi, A.; Salvadego, D.; Da Ponte, A.; Bertin, N.; Dardis, A.; Cattarossi, S.; Devigili, G.; Reccardini, F.; Bembi, B.; Grassi, B. Investigation on acute effects of enzyme replacement therapy and influence of clinical severity on physiological variables related to exercise tolerance in patients with late onset Pompe disease. Neuromuscul. Disord. 2017, 27, 542-549. [CrossRef]

27. Marzorati, M.; Porcelli, S.; Reggiori, B.; Morandi, L.; Grassi, B. Improved exercise tolerance after enzyme replacement therapy in pompe disease. Med. Sci. Sports Exerc. 2012, 44, 771-775. [CrossRef] [PubMed]

28. Banugaria, S.G.; Prater, S.N.; Patel, T.T.; Dearmey, S.M.; Milleson, C.; Sheets, K.B.; Bali, D.S.; Rehder, C.W.; Raiman, J.A.; Wang, R.A.; et al. Algorithm for the early diagnosis and treatment of patients with cross reactive immunologic material-negative classic infantile pompe disease: A step towards improving the efficacy of ERT. PLoS ONE 2013, 8, e67052. [CrossRef] 
29. Prater, S.N.; Patel, T.T.; Buckley, A.F.; Mandel, H.; Vlodavski, E.; Banugaria, S.G.; Feeney, E.J.; Raben, N.; Kishnani, P.S. Skeletal muscle pathology of infantile Pompe disease during long-term enzyme replacement therapy. Orphanet J. Rare Dis. 2013, 8, 90. [CrossRef]

30. Kuperus, E.; Kruijshaar, M.E.; Wens, S.C.A.; De Vries, J.M.; Favejee, M.M.; Van Der Meijden, J.C.; Rizopoulos, D.; Brusse, E.; Van Doorn, P.A.; Van Der Ploeg, A.T.; et al. Long-term benefit of enzyme replacement therapy in Pompe disease: A 5-year prospective study. Neurology 2017, 89, 2365-2373. [CrossRef]

31. Chien, Y.H.; Tsai, W.H.; Chang, C.L.; Chiu, P.C.; Chou, Y.Y.; Tsai, F.J.; Wong, S.L.; Lee, N.C.; Hwu, W.L. Earlier and higher dosing of alglucosidase alfa improve outcomes in patients with infantile-onset Pompe disease: Evidence from real-world experiences. Mol. Genet. Metab. Rep. 2020, 23, 100591. [CrossRef] [PubMed]

32. Case, L.E.; Bjartmar, C.; Morgan, C.; Casey, R.; Charrow, J.; Clancy, J.P.; Dasouki, M.; DeArmey, S.; Nedd, K.; Nevins, M.; et al. Safety and efficacy of alternative alglucosidase alfa regimens in Pompe disease. Neuromuscul. Disord. 2015, 25, 321-332. [CrossRef] [PubMed]

33. Van Gelder, C.M.; Poelman, E.; Plug, I.; Hoogeveen-Westerveld, M.; van der Beek, N.A.M.E.; Reuser, A.J.J.; van der Ploeg, A.T. Effects of a higher dose of alglucosidase alfa on ventilator-free survival and motor outcome in classic infantile Pompe disease: An open-label single-center study. J. Inherit. Metab. Dis. 2016, 39, 383-390. [CrossRef] [PubMed] 